Poster

\title{
Utilization, Reliability, and Validity of a Smartphone App for Chronic Pain Management: A Randomized Controlled Trial
}

\author{
Robert N Jamison, PhD; Edgar L Ross, MD \\ Department of Anesthesiology, Brigham and Women's Hospital, Harvard Medical School, Chestnut Hill, MA, United States
}

\section{Corresponding Author:}

Robert N Jamison, PhD

Department of Anesthesiology

Brigham and Women's Hospital

Harvard Medical School

Suite 320

850 Boylston Street

Chestnut Hill, MA, 02467

United States

Phone: 16177329046

Fax: 16177329050

Email: rjamison@partners.org

\section{Abstract}

Background: There has been an explosion of smartphone applications (apps) that have been used to track health data and change the management of chronic diseases. However, there have been very few studies designed to comprehensively examine the usability, acceptability, reliability, utility, and content and face validity of a smartphone pain app.

Objective: The overall aim of this study was to determine the effect of introducing a smartphone pain app, for both Android and iPhone devices, that enables chronic pain patients to assess, monitor, and communicate their status to their providers.

Methods: This study recruited 105 chronic pain patients to use a smartphone pain app. The patients were randomized to either receive 2-way messaging or a standard message on the smartphone app using a stratified randomization table. Those in the experimental group $(n=53)$ received 2-way messaging of weekly supportive text messages and feedback about their progress by the study research assistant (eg, "Hello Dave! It looks like your pain, mood and activity interference this week have improved way to go!"). Those in the control group ( $n=52)$ received a standard reply of "Thank you. Your message has been received" every time the participants sent a message through the app. All subjects completed baseline measures and were asked to record their progress every day for 3 months, with the opportunity to continue for 6 months. All participants were supplied a Fitbit to track daily activity. Summary line graphs were posted to each of the patients' electronic medical records, and physicians were notified of their patients' progress.

Results: Ninety patients successfully downloaded the pain app. Average age of the participants was 47.1 (range 18-72), 63.8\% were female, and $32.3 \%$ reported multiple pain sites. Adequate validity and reliability was found between the daily assessments and standardized questionnaires $(r=0.50)$ and in repeated daily measures ( $r=0.69$ pain; $r=0.83$ sleep). Patient satisfaction survey results showed that the app was easy to use and easy to navigate, and those subjects with more daily assessments were found to be more satisfied with the app compared with those who used the app less often $(P<.05)$. Those patients assigned to the 2 -way messaging condition on average tended to use the app more and submit more daily assessments (95.6 vs 71.6 entries) and found the app more appealing, easier to use and to navigate, and less bothersome than those without the 2-way messaging ( $P<.05)$, but differences between groups in adherence to the pain app over time were not significant. Seven pain management physicians and six pain fellows completed an anonymous satisfaction survey at the end of the trial. A total of $85.7 \%$ reported being satisfied with the way the app was used in the clinic and liked receiving the pain app summary messages. Also, $85.7 \%$ believed that using the app would improve their overall practice, while none of the physicians felt that the pain app was an added burden to the clinic.

Conclusions: This study highlights some of the benefits and challenges in utilizing smartphone apps to manage chronic pain patients, and provides insight into those individuals who might benefit from mHealth technology. Overall, the smartphone pain app was found to be usable, valid, reliable, and easily accepted among patients and providers alike. The 2-way messaging feature was also found to moderately improve compliance with daily assessments. Mobile application technologies possess advantages 
and possibilities that have not previously existed and future programs are needed that tailor to the needs of the individual to engage and motive the user to make changes that enhance health care management.

(iproc 2016;2(1):e20) doi: 10.2196/iproc.6060

\section{KEYWORDS}

chronic pain; innovative technology; pain app; mHealth; smartphone

This poster was presented at the Connected Health Symposium is displayed as an image in Figure 1 and as a PDF in Multimedia 2016, October 20-21, Boston, MA, United States. The poster Appendix 1.

Figure 1. Poster.

\section{Utilization, Reliability and Validity of a Smartphone App for Chronic Pain Management:A Randomized Controlled Trial}

Robert N. Jamison, Ph.D., Edgar L. Ross, M.D.

Pain Management Center, Dept of Anesthesiology, Brigham and Women's Hospital, Harvard Medical School, Chestnut Hill, MA, 02467 USA
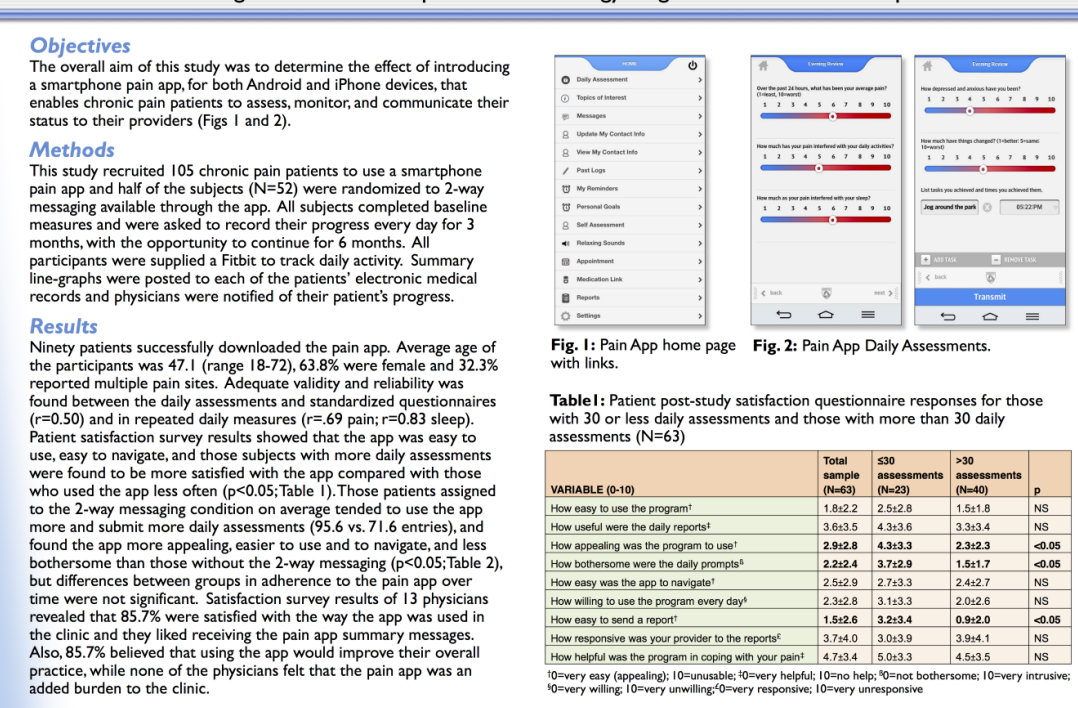

Fig. I: Pain App home page Fig. 2: Pain App Daily Assessments.

with links.

Tablel: Patient post-study satisfaction questionnaire responses for those with 30 or less daily
assessments $(N=63)$

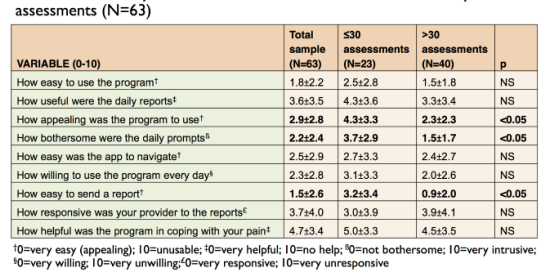

Table 2: Patient post-study satisfaction questionnaire responses for those with 2-way messaging (experimental) and those without 2-way messaging (controls; $\mathrm{N}=63$ )

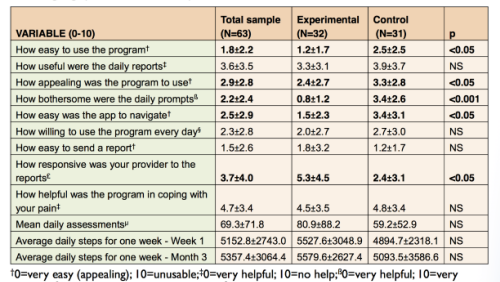

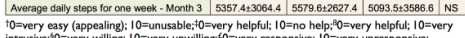

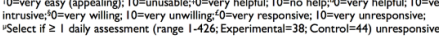

Discussion

This study highlights some of the challenges and benefits in utilizing smartphone apps to manage chronic pain patients, and provides insigh into those ind viduals who might benefit from $m$ Health technology. reliable, and easily accepted among patients and providers alike. The 2-way messaging feature was also found to moderately improve compliance with daliy assessments. Mobile application technologies possess advantages and possibilties that have not previously existed
and future studies are needed to address the best ways that mobile technologies might enhance health care management

Acknowledgement: A pilot granc from the Mayday Fund and a government grant
through the Center for Future Technologies in Cancer Care (through Boston University

\section{Multimedia Appendix 1}

Poster.

[PDF File (Adobe PDF File), 1MB-Multimedia Appendix 1]

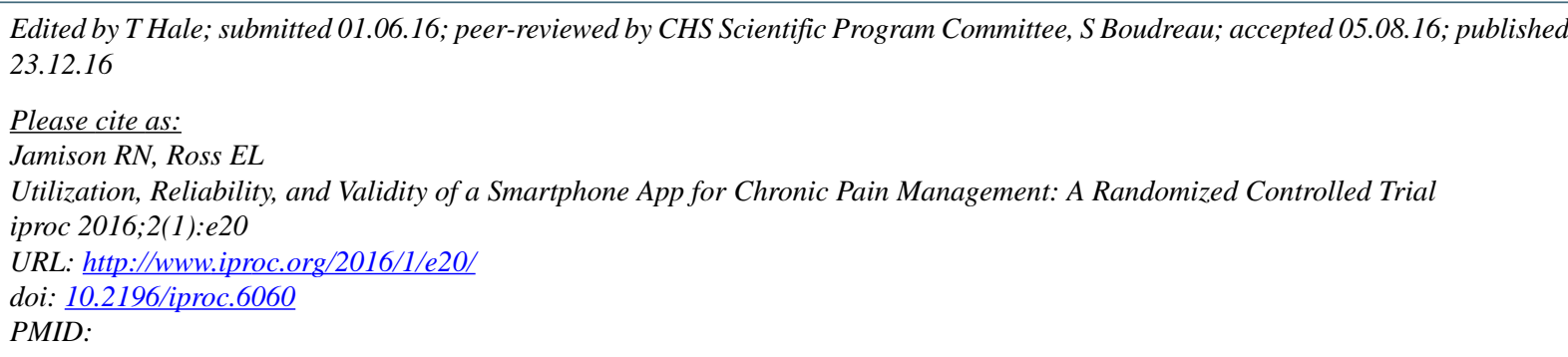

CRobert N Jamison, Edgar L Ross. Originally published in Iproceedings (http://www.iproc.org), 23.12.2016. This is an open-access article distributed under the terms of the Creative Commons Attribution License (http://creativecommons.org/licenses/by/2.0/), which permits unrestricted use, distribution, and reproduction in any medium, provided the original work, first published in 
Iproceedings, is properly cited. The complete bibliographic information, a link to the original publication on http://www.iproc.org/, as well as this copyright and license information must be included. 